\title{
The internal dynamics of stellar clusters
}

\author{
Christian M. Boily \\ Observatoire astronomique de Strasbourg \& CNRS UMR 7550 \\ 11, rue de l'université, Strasbourg F-67000, France \\ email: christian.boily@astro.unistra.fr
}

\begin{abstract}
In this brief review I summarise recent progress in the area of stellar dynamics, focusing on the dynamics of bound, self-gravitating stellar associations in isolation and (approximate) equilibrium. The basics of stellar dynamics are first outlined and the importance of stellar evolution is stressed. Subsequently, I argue that the evolution of anisotropic clusters of stars still holds solutions to current outstanding problems, such as the dynamics of galactic nuclei. I take a more personal standpoint when discussing the role of stellar evolution in the dynamics on relaxation timescales and draw from several recent models to underscore that a major step forward has been made in coupling stellar evolution and dynamics. I then briefly visit the issue of multiple stars and highlight some as yet unsolved problems.
\end{abstract}

Keywords. galaxies: star clusters, binaries: general

\section{Introduction}

It has been a long while since Newton wrote down the basic equations that govern the evolution of self-gravitating systems of point sources. That a general, exact solution to the problem of evolving a set of $N$ mass elements over time will forever elude us must have come as a shock to the pioneers of stellar dynamics, who realised that progress in this field would come only through a statistical approach to constructing observables derived from theory. That long-ish statement can be rephrased as nonlinearity rules in Newtonian stellar dynamics, and so one must resort to statistical methods to solve for the dynamics of individual clusters (mainly drawn from a Fokker-Planck treatment of the equations) or, from averaging over large ensembles of models to smooth over singular solutions that could be attributed to the choice of initial conditions.

In this short review, I will take up the last point, but start with general considerations and the point-mass treatment of stellar clusters. I will then highlight what has been learned from evolving models with inclusion of a mass spectrum and stellar evolution. The single stellar evolution approach has been extended to multiple stars (binaries and hierarchical higher-order multiples), which are an essential ingredient to understand the demographics of exotic stellar remnants (pulsars, blue stragglers and the like). I will end with an overture to ongoing efforts by modellers to include ever more detailed physics in their calculation but with a view to 'packaging' software for the benefit of the broader community. The scope of this paper is narrow and not much will be found here by those seeking updates on observational campaigns and techniques: those aspects are covered in depth in the recent conference proceedings by Richtler \& Larsen (2009) and Vazdekis \& Peletier (2007). Constraints of time and space (and skills) mean that I have to select what instinctively seems more in tune with the spirit of the meeting in Rio. More substantial recent surveys of the field start with the well-balanced Meylan \& Heggie (1997) review and include, among many others, several excellent contributions in Vesperini et al. (2008). 


\section{Basics}

It will help to follow a thread of increasing complexity when discussing stellar cluster dynamics. Let me recall that the problem of retracing orbits in a self-gravitating system of identical point sources, satisfying the scalar virial theorem

$$
\sigma^{2} \equiv-\frac{G M}{r_{\mathrm{g}}}
$$

(where $\sigma$ is the average squared velocity dispersion, $M$ the total mass and $r_{\mathrm{g}}$ the gravitational radius: not equal to the truncation radius, in general), boils down to two timescales: one measuring the time needed to cross a nominal volume enclosed by the truncation radius, and the other the time required for kinetic energy to diffuse effectively through the system by repeated two-body interactions. Since we are focussing on the internal cluster dynamics, it is convenient to introduce the (spherical) half-mass radius $r_{\mathrm{h}}$ and define the crossing time thus

$$
t_{\mathrm{cr}} \equiv \frac{2 r_{\mathrm{h}}}{\sigma}
$$

and note that I make no distinction between the one- and three-dimensional velocity dispersion because the definition of $t_{\mathrm{cr}}$ is not unique and this issue is not important here. This is clear from the definition of the two-body relaxation time, $t_{\mathrm{r}}$, of Meylan \& Heggie (1997), which features $r_{\mathrm{g}}$ :

$$
t_{\mathrm{r}} \equiv 0.138\left(\frac{r_{\mathrm{h}}}{2 r_{\mathrm{g}}}\right)^{3 / 2} \frac{N}{\ln (0.4 N)} t_{\mathrm{cr}} .
$$

In the latter equation, the numerical factor in front of $t_{\mathrm{cr}}$ is not fixed by mass (i.e., particle number) alone, but rather includes a dimensionless ratio $r_{\mathrm{h}} / r_{\mathrm{g}}$, which together with $N$ encompasses the basic physics that drives analysis: whenever the ratio $t_{\mathrm{r}} / t_{\mathrm{cr}} \gg 1$, we can treat the system as being in near equilibrium and in the regime of slow evolution. It is remarkable that profiling of the mass in space only enters in the considerations through $r_{\mathrm{h}} / r_{\mathrm{g}}$. In the regime of slow evolution, one aims to solve the Boltzmann equations, suitably rewritten with Rosenbluth potentials to account for the collisional terms. This is the 'large- $N$ ' limit, when a distribution function is a sensible representation of the background mean potential. Formally, one writes

$$
\frac{\mathrm{d} f(\boldsymbol{r}, \boldsymbol{v}, t)}{\mathrm{d} t}=\left(\frac{\partial f(\boldsymbol{r}, \boldsymbol{v}, t)}{\partial t}\right)_{\text {coll }}
$$

and expands the right-hand side using diffusion coefficients to evolve the distribution function, $f$, through phase space (e.g., Spitzer 1987). An appealing variant of this approach is to consider the diffusion coefficients as statistical weights for a two-body encounter leading to an exchange of kinetic energy: this is the road to Monte Carlo modelling. Now the core-halo structure of star clusters implies shorter dynamical times in the core and therefore more efficient diffusion there than in the outer envelope. This leads to core collapse, as the envelope drains potential energy from the core (by expanding) which loses mass and shrinks. Both Fokker-Planck and gas-dynamical solutions (treating the flow of mass in the continuous-fluid limit) have long confirmed theoretical expectations already developed in the 1960s of the irreversibility of this process, once the core density exceeds $709 \times$ the cluster average density (Cohn 1979; Lynden-Bell \& Eggleton 1980). It may seem far-fetched to treat the decoupled collapsing core from a distribution-function perspective given that the dense core drops to zero size and mass in a finite time: clearly, methods based on distribution functions are aimed at massive, rich clusters: see Aarseth 
\& Heggie (1998) for a discussion. The potential mapped by a small number of stars confined to the core of a cluster becomes ever more granular and no longer amenable to a fluid approach. Indeed, small- $N$ systems depart from spherical symmetry (through Poissonian noise) and so the fine details of the evolution of the cluster in the final stages of core collapse derived from Fokker-Planck modelling become more uncertain. This is where $N$-body modelling become essential, as it supplements other approaches to stellar dynamics through more general three-dimensional coding of the equations of motion. The caveat, of course, is that to date the largest- $N$ calculations performed are limited to $N \sim 10^{5}$ (e.g., Hemsendorf \& Merritt 2002; Baumgardt et al. 2003, 2008; Fujii et al. 2008 ) which is still below the average of $\simeq 4.3 \times 10^{5}$ for Galactic clusters (assuming a standard stellar mass function; Meylan \& Heggie 1997) and further still from the large- $N$ regime sought. It is reassuring to find that the simple problem of core collapse for equalmass systems over a time $t \simeq 15 t_{\mathrm{r}}$ is well reproduced by completely different methods (e.g., Takahashi 1995; Makino 1996; Baumgardt et al. 2003; Spurzem et al. 2005).

Against this backdrop, let me chart the few issues that I will address in the remainder of this paper. Once more, my leitmotiv is the complexity of systems in terms of the physics that they include. I divide these into four broad classes of problems that are of interest here, which are organised according to whether or not one includes stellar evolution (SE) in the calculations and whether or not multiple stars are included in the initial conditions. Needless to say, the combination of multiple stars with full stellar evolution (including mass exchange, etc.) is the more challenging case to theorists. Below, I start with the point-mass dynamics of single stars.

\section{Point-mass dynamics}

\subsection{Single stars with anisotropy}

Surely, one should be content with the outline of Section 2 above, as this is a wellbeaten path. Not quite. One problem still causing headaches is to deconvolve projected data while relaxing the hypothesis of isotropy. Recall that two-body relaxation tends to make the central region of clusters isotropic, while the outer expanding envelope is more and more anisotropic as a result of excess radial motion. Louis \& Spurzem (1991), Takahashi (1995) and Drukier (1999) discussed the Fokker-Planck evolution towards core collapse of initially anisotropic clusters (see Giersz \& Spurzem 1994 for an earlier similar study of gas-dynamical models). These models were all nonrotational and while evolution proceeded roughly as for the isotropic models, Takahashi (1995) reported delayed core collapse, from 15 to $17.6 t_{\mathrm{r}}$, which may be a source of scatter in comparative photometric surveys of cuspy clusters (see, e.g., Noyola \& Gebhardt 2006 for a sample of 38 clusters from HST archive data). The importance of anisotropy was stressed in an application to the G1 cluster in M31, where excellent fits to observed parameters (both kinematics and photometry) were recovered from merging two smaller clusters (Baumgardt et al. 2003). Such an interpretation has been challenged by Gebhardt et al. (2005) based on higherresolution photometric data indicating a profile best adjusted with a $M_{\mathrm{bh}} \simeq 20000 \mathrm{M}_{\odot}$ central black hole. Pooley \& Rappaport (2006) and Kong (2007) pointed out that X-ray data could provide confirmation of the presence of a black hole, if angular resolution could pin down the source well enough to rule out alternatives based on a hypothetical population of X-ray binary sources (for example). Whatever the ultimate outcome of these investigations, the point was not lost that anisotropy has to be factored in when exploring the dynamics of clusters at the most basic level.

But the yet more important effect attributable to anisotropy must be systematic streaming, which leads to net rotation and strong deviations from spherical symmetry. 
That Galactic clusters such as $\omega$ Cen and 47 Tuc are rotating has been known for a long time and ideas were already put forward many years ago to include rotation in the models (e.g., Agekian 1958; Shapiro \& Merchant 1976; Hachisu 1979). Still, it is only relatively recently that self-consistent two-dimensional Fokker-Planck modelling with distribution functions of the form $f\left(E, L_{z}\right)$ was introduced by Einsel \& Spurzem (1999), who confirmed Hachisu's gravo-gyro catastrophe [see also Ernst et al. (2007) and Kim et al. (2008) for investigations of this problem with $N$-body integrations]. The inclusion of rotation speeds up core-collapse in single-mass cluster dynamics, by a multiplicative factor of up to $\approx 3$ compared to isotropic models. Interestingly, projected quantities derived from these models have only been made available relatively recently by Fiestas et al. (2006), $\dagger$ who ran a grid of models with an improved version of Einsel's original Fokker-Planck code. As in the Baumgardt et al. work on anisotropic clusters resulting from the merging of two clusters, and hence quite possibly residual rotation from that event, one should hope that rotation will, in the future, become an integral part of the parameter space explored to fit data. Some work is underway to embed black holes in clusters with rotation and it will be interesting to monitor the outcome of such models in applications to clusters such as G1 or M15 (see Fiestas et al. 2008 for preliminary work).

\subsection{Chaos and sensitivity to the initial configuration}

The orbit integration of the collisional $N$-body problem is known to be sensitive to the initial configuration of the system, in the sense that small deviations in the coordinates of a body are amplified exponentially with time at the onset of integration. To track this issue, Miller (1971) set up a variational method to monitor the orbits of massless bodies, given a small shift in phase space with respect to actual massive bodies that define the potential. The game consists of measuring the distance $\Delta$ between orbits traced out by the massless bodies to that of the body nearest to them in the initial configuration. Linear perturbation analysis tells us that $\Delta$ grows exponentially with time. One's instinct is that the $e$-folding time should become longer with increasing $N$ as one moves closer to the idealised limit of the smooth (and static) mean field for which orbits must satisfy the collisionless Boltzmann equation (CBE; see Binney \& Tremaine 2008). Detailed analysis indicates instead that the $e$-folding time, $t_{e}$, decreases with $N$, a trend driven mainly by accrued small-scale two-body scatter (Goodman et al. 1993). Hemsendorf \& Merritt (2002) confirmed this expectation with a series of $N$-body calculations with $N$ running from 100 to $\approx 132000$, and obtained a rough fit for $N \gtrsim 32000$ of the form (see their figure 2)

$$
t_{e}=\frac{0.12 t_{\mathrm{cr}}}{\ln 0.7 \ln N}
$$

with a notable weak dependence on $N$. Hut \& Heggie (2002) argue that the exponential growth saturates at a few $\times t_{e} \propto t_{\mathrm{cr}}$. Their analytical approach, backed up by numerical experiments, leads to a turnover, from an exponential, to a power-law growth rate over a relaxation time. Since two diverging paths will have, sensibly, the same potential energy but different kinetic energy, this growth can be expressed as a relative shift in total energy,

$$
\frac{\delta E}{E} \propto\left(\frac{t}{t_{\mathrm{r}}}\right)^{\frac{1}{2}} .
$$

This near-saturation has two immediate consequences for the purpose of tracing orbits numerically: (i) it implies that the orbits can be known up to a finite precision, beyond

$\dagger$ See http://www.ari.uni-heidelberg.de/clusterdata/ 
which interpretation becomes unphysical and (ii) it sets a constraint on the level of fluctuations that can be 'spontaneously' transmitted from the background potential, to a multiple star (say, a binary or triple star).

Point (ii) is best left for Section 5. Point (i) is easier to understand if we carry out a Gedankenexperiment such that after a crossing time of evolution (say), the phase-space coordinates are synchronised and a restart performed. If the data were synchronised to arbitrary accuracy by interpolation of the known positions and velocities, that precision would be lost after $\sim t_{\text {cr }}$ of integration through exponential growth of rounding errors. That remark can be turned on its head, by saying that when coordinates are made synchronous, the procedure can tolerate less-than-perfect accuracy and be equally valid for a restart (as long as one uses an integration time well exceeding a crossing time). Beyond issues of recovering accurate data for the sake of dissecting the orbits of a model cluster, one is confronted with the problem of data storage associated with a core-halo structure. In such a system, the dynamical time $t_{\mathrm{cr}}$ varies significantly: for a rich cluster, one may compute $t_{\mathrm{cr}} \sim 10^{3}$ years in the core, and $t_{\mathrm{cr}} \sim 10^{6}$ years in the halo. Clearly, resolving orbits everywhere requires a large throughput in the core, and a much reduced one in the halo. But synchronicity required over the whole system to construct snapshots translates to huge data sets. Practitioners have long realised that a self-respecting integrator would negotiate orbits more carefully where needed, by adopting a smaller time step (the block time step procedure in conjunction with the Hermite integration scheme is the standard of choice; McMillan 1986; Makino \& Aarseth 1992; see Aarseth 2003 for more background). A major space-saving procedure is to dump phase-space coordinates at a fixed number of integration steps (instead of equal time intervals) and reconstruct the orbit using the same scheme for integration to interpolate to the desired time (e.g., Faber et al. 2009). It is not clear how to translate the precision achieved using this approach to requirements that meet the same level of tolerance as what is achieved by the divergence in energy over a crossing time (Hut \& Heggie 2002), although one can guess that the optimal approach (to orbit reconstruction) factors in the uncertainty associated with the reference orbit.

I will now show that only statistics can save us from compiling heaps of data only to follow a single-mass cluster up to core collapse. The classical solution has the core radius going to zero at a time $t \equiv t_{\mathrm{c}}$. Prior to that instant, the core radius $\propto\left(t_{\mathrm{c}}-t\right)^{2 /(6-\alpha)} \rightarrow 0$ as $t \rightarrow t_{\mathrm{c}}$ (the constant $\alpha \approx 2.21$; e.g., Lynden-Bell \& Eggleton 1980; see Spitzer 1987). The mass fraction of stars confined to the core $M_{\mathrm{c}} \propto\left(t_{\mathrm{c}}-t\right)^{(6-2 \alpha) /(6-\alpha)} \propto\left(t_{\mathrm{c}}-t\right)^{0.42}$ drops rapidly as $t \rightarrow t_{\mathrm{c}}$, however the central density $\rho_{\mathrm{c}} \propto\left(t_{\mathrm{c}}-t\right)^{-2 \alpha /(6-\alpha)} \propto\left(t_{\mathrm{c}}-t\right)^{-1.16}$ diverges to the same limit, which in turn implies that the orbital periods of stars near the centre $\propto 1 / \sqrt{G \rho_{\mathrm{c}}} \propto\left(t_{\mathrm{c}}-t\right)^{0.58}$ also drop to zero. But owing to the Lagrangian nature of the Hermite scheme, the volume of data stored over one revolution remains constant. As a result, the net volume of data scales with $\propto M_{\mathrm{c}} \times \sqrt{G \rho_{\mathrm{c}}} \propto\left(t_{\mathrm{c}}-t\right)^{-0.16}$ and therefore increases as a weak power of time. The singularity as $t \rightarrow t_{\mathrm{c}}$ will not be attained in practice owing to the formation of binary stars by three-body interactions (the probability $\propto \rho^{2} \times \rho r_{\mathrm{c}}^{2} \sigma \propto\left(t_{\mathrm{c}}-t\right)^{-2.92}$ is a steep function of a vanishing argument). Nevertheless, the trend with time will be real and may require a fix in the form of a coarser sampling in the later phases of a cluster's evolution.

\subsection{Single stars with a mass spectrum}

The dynamics of point-mass clusters becomes more entertaining once a spectrum of masses is included. The most immediate effect is the segregation of stars (by mass) through a process which Spitzer termed the asymptotic tendency towards equipartition of kinetic energy: since this is driven by two-body interactions, one finds that segregation 
operates on the relaxation time $t_{\mathrm{r}}$, but for a multiplicative factor that accounts for the spread in the mass spectrum of the point masses (Spitzer 1969; Meylan \& Heggie 1997):

$$
t_{\mathrm{seg}} \simeq \frac{\langle m\rangle}{\max \{m\}} \frac{\bar{\rho}}{\rho_{\mathrm{h}}}\left(\frac{r_{\mathrm{h}}}{r_{\mathrm{g}}}\right)^{3 / 2},
$$

where $\langle\ldots\rangle$ denotes ensemble averaging, and overbar mass-averaged quantities, and I have taken an estimate of $t_{\mathrm{seg}}$ at the half-mass radius where the density $\rho\left(r_{\mathrm{h}}\right)$ is evaluated. Note that $t_{\text {seg }}$ presumes that each mass element in the set $\left\{m_{i}\right\}$ is constant, so that one expects the initially more massive stars to orbit closer to the centre than less massive bodies. The hierarchy of masses would also define a hierarchy of $t_{\text {seg }}$ times, using the same definition (3.3) but replacing the maximum mass by the one of interest.

In practice, an application of Equation (3.3) only makes sense if the segregation time is shorter than the lifetime of the zero-age main sequence stars of the same initial mass (metals are not taken into account for the moment). Several authors have bounced on this observation to deduce that massive stars converging near the centre of a cluster would inevitably dissipate tidal energy through their envelope or by a direct hit, coalesce and then possibly form a black hole (e.g., Portegies Zwart \& McMillan 2002; Portegies Zwart et al. 2004; Gürkan et al. 2004; Freitag et al. 2006a,b; Ardi et al. 2008; Gaburov et al. 2008). Therefore, mass segregation would provide a natural setup to trigger the formation of very massive stars or even a black hole in any sufficiently rich and dense cluster at birth (with a full spectrum of stellar masses). The implications for current and future gravitational waves from inspiraling black holes and their detection are clear (see Fregeau et al. 2006; Amaro-Seoane et al. 2007).

There are several issues related to the formation of a black hole and the migration of stars in its neighbourhood. Spitzer (1969) realised that when two populations of stars cohabit in a cluster, the trend towards equipartition leads to the more massive component decoupling from the other, lighter component to form a (quasi-) self-bound core. This occurs in two-population systems of masses $m_{1}, m_{2}$, with $m_{2}>m_{1}$, whenever the ratio of total mass in each group satisfies

$$
\frac{M_{2}}{M_{1}}<\beta\left(\frac{m_{1}}{m_{2}}\right)^{3 / 2},
$$

where the dimensionless constant $\beta \approx 0.16$. This relation goes a step further than Equation (3.3), which does not take into consideration the self-gravity of the most segregated group. Now, in first instance one would like to know what degree of segregation a cluster can reach, and whether Equation (3.4) will take it back to the evolutionary track of singlemass clusters and core collapse (which involves the innermost region only). Gaburov et al. (2009) and Glebbeek et al. (2009) pointed out that a star orbiting in the core of a dense cluster may undergo several collisions, leading to fusion once dissipative effects are taken into account. Paramount to understanding how this will bear on observations is the state of equipartition of the system at the onset of evolution (be it numerical modelling or otherwise). Several observations point to clusters being segregated at birth (e.g., de Grijs et al. 2002; McCrady et al. 2005), while theoretical studies now seem to favour segregation taking place at the very formation of a cluster (mainly by scattering of substructures: McMillan et al. 2007; Allison et al. 2009; Vesperini et al. 2009). Baumgardt et al. (2008) and Decressin et al. (2008) explore some observational consequences of early mass segregation. A crucial result from theory is that mass segregation in clusters with rotation proceeds in a nontrivial way, on a timescale that optimises angular-momentum diffusion (see Kim et al. 2004 for details). Here, I mention an application to galactic nuclei where 
multimass models have drawn mainly from Monte Carlo simulations of dense environments (Fregeau et al. 2002; Freitag et al. 2006b), while $N$-body modelling has focussed on the orbital migration of black-hole binaries or the interplay between single-star populations and black-hole binaries (see Berentzen et al. 2009 for a recent update on this problem). An important distinction with respect to stellar clusters is the inclusion of a massive black hole in the calculations at rest at the centre of the system. The segregation time (Equation 3.3) may yet be shorter than a massive-star lifetime, $\tau$, owing to the high density of light stars around the black hole (Freitag et al. 2006a). In that case, the massive stars will sink to the centre rapidly and hit the stalling radius (Merritt 2006), where the orbits are quasi-Keplerian. At that point, little evolution was expected from star-star scattering, an issue revisited recently by Alexander \& Hopman (2009), who introduced the dimensional parameter $\Delta$ (rewritten in the notation of Equation 3.4),

$$
\Delta \equiv 4 N_{2} M_{2}^{2} /\left[N_{1} M_{1}^{2}\left(3+M_{2} / M_{1}\right)\right] .
$$

$\Delta>1$ for self-scattering among massive stars, whereas $\Delta<1$ for a depleted sample of massive stars scattering off background light stars (here of mass $m_{1}$ ). An interpretation of this result is that massive stars may yet enhance their own scattering to reach below the stalling radius. It will be interesting to derive observational consequences of this possibility since the profile of massive bodies will differ from that of background stars: spatial gradients in spectroscopic indices would then indicate whether effectively $\Delta>1$ in nuclei (see Alexander \& Hopman 2009 for a discussion of this possibility). A most interesting test will be the Milky Way centre, where massive S stars are known to orbit within the stalling radius, a notoriously difficult puzzle to solve using the principle of stellar migration alone (see, e.g., Merritt et al. 2009 or Portegies Zwart et al. 2003 for discussions of the migration of clusters with intermediate-mass black holes in their midst).

\section{Dynamics with stellar evolution}

There have been great advances since 2001 in coupling dynamics with stellar evolution tracks, in large part driven by joint efforts such as MoDeST (Hut 2003) and MUSE (Portegies Zwart et al. 2009). Below. I revisit some outstanding issues and prospects for future developments.

\subsection{Chronicle of a dissolution foretold}

The basic problem with coupling stellar evolution and dynamics is that the stars lose mass at the same time as their luminosity varies rapidly, which makes the construction of observables a tad more tricky (all the more so with small-ish clusters for which broadband photometry may be dominated by a few bright stars). At a more basic level, I note that stellar mass loss induces a steady drift in the potential not accounted for in the dynamics of fixed point-mass elements. To illustrate this, I write the fully dimensional Newtonian-dynamics equations for a body of mass $m$, which is an explicit function of time,

$$
\frac{\mathrm{d} \boldsymbol{p}}{\mathrm{d} t}=m \dot{\boldsymbol{v}}+\dot{m} \boldsymbol{v}=-m \boldsymbol{\nabla} \phi(\boldsymbol{r}, t),
$$

with $\boldsymbol{p}$ the linear momentum and other quantities assume their usual meaning. If I now specialise to the case of spherical symmetry, I can write for the density $\rho(r, t) \simeq$ $m(t) / m(0) \rho(r, 0)$ if all stars have equal mass and the structure of the cluster has changed little over $t \sim t_{\mathrm{cr}}$, and $m(t)$ is some decreasing function of time. The same will be true of $\phi(r, t)$ in terms of $\phi(r, 0) \equiv \phi_{o}$ as for the density because of Poisson's equation. One 
may thus compute the rate of change of the binding energy of any star in the system as

$$
\dot{E}=\dot{m} \phi+m \boldsymbol{v} \cdot \nabla \phi+m \partial_{t} \phi++m \boldsymbol{v} \cdot \dot{\boldsymbol{v}}+\frac{1}{2} m v^{2} \simeq-\dot{m}\left[\frac{1}{2} v^{2}-2 \frac{m(t)}{m(0)} \phi_{o}\right],
$$

where the last step follows from the 'frozen potential' approximation. The key insight from Equation (4.2) is that $\dot{E}>0$ for any initially bound orbit since $\dot{m}<0$ by hypothesis. If we imagine that the cluster is so evolved that the self-similar core-collapse flow applies approximately to the inner region, then it is easy to show that the binding energy of the decoupled core $W_{\mathrm{c}} \propto G M_{\mathrm{c}}^{2} / r_{\mathrm{c}} \propto\left(t_{\mathrm{c}}-t\right)^{0.31}$ drops to zero (along with its mass) at a rate $\dot{W}_{\mathrm{c}} \propto\left(t_{\mathrm{c}}-t\right)^{-0.69}$. But since the net effect of mass loss through stellar winds is to pump in energy $(\mathrm{d} E / \mathrm{d} t>0)$, there must come a time at which the binding energy of the core is positive for a finite core mass and radius. This argument is correct for any orbit confined to a small region around the cluster centre when mass loss begins. Since these are generally the most bound orbits, one is drawn to the conclusion that stellar winds must severely disrupt the classical self-similar solution by applying brakes to core contraction. Whether this occurs before the formation of binaries by three-body encounters, which would also halt collapse, depends on the ratio $t_{\mathrm{r}} / \tau$ of cluster relaxation time to main-sequence lifetime, $\tau$. For the problem at hand, we must understand that the answer is a strong function of the cluster's initial conditions and stellar spectral type. One thing is clear, though, the systematic inclusion of stellar evolution in the modelling has had a deep impact on our understanding of these objects: below I track two areas that have benefited much from this, mass estimation of extragalactic clusters, and dynamics of multiple star populations.

\subsection{Chronicles of a mass not so well foretold}

The virial theorem is a powerful tool when applied to averaged properties of a selfbound cluster (at least for clusters of age > 30 Myr or so; see Bastian \& Goodwin 2006; de Grijs \& Parmentier 2007). What is less clear is whether one may presume that the age of such young clusters inevitably is $\ll t_{\mathrm{r}}$ or indeed the equipartition timescale $t_{\text {seg }}$. Theoretical modelling is a way of recovering a conversion factor (I will call it $\eta$ ) from mock spectrophotometric data of the projected models. Dimensional analysis alone gives

$$
\eta \equiv \frac{r_{\mathrm{ph}} \sigma_{\mathrm{s}}^{2}}{G M}
$$

where $r_{\mathrm{ph}}$ is some photometric radius, $\sigma_{\mathrm{s}}$ a spectroscopic one-dimensional velocity dispersion and $M$ the total mass of the model. Under virial equilibrium conditions, one finds $\eta \simeq 8$ for a good range of King models. The catch is that while $\eta$ may remain constant for mass-weighted quantities, in reality flux-weighted quantities will evolve on the same or a similar timescale as the massive stars for several important cases (see Boily et al. 2005; Fleck et al. 2006 for a fuller exploration of single-star models). As a result, $\eta$ may well vary by a factor of up to three in the case of single-star populations. Note that the idea of converting light to mass is used by observers, for instance see McCrady \& Graham (2007) or Östlin et al. (2007), yet theorists have been slow to convert their model data into observable quantities. Some work has attempted to draw consequences for full globular cluster mass functions by deconvolving the mass of model clusters of a given initial mass and age (Fleck et al. 2006; Kruijssen \& Portegies Zwart 2009), although much remains to be done in this area given the oversimplification of the stellar populations modelled. Yet, it is clear that $\eta$ is a key parameter to evaluate as it may hold the answer to cluster survival and the true mass function (de Grijs \& Parmentier 2007). 


\subsection{Chronicled desperation, or dynamics with multiple stars...}

One of the main reasons for the poor situation with $\eta$ is surely that an analytical treatment is impossible, and that high-resolution numerical models are expensive computationally, regardless of the method used. As a consequence, ensemble-averaged quantities are even more problematic to secure. A notable difficulty is that multiple stars complicate the issue even more.

Consider a cluster of mixed stellar populations, of single and binary stars. Surely, if the binary population represents only a small percentage of all stars, it can carry little of the total light and mass. In that case, one would treat binaries to zeroth order as (on average) stars twice as massive as the others and derive observables from that perspective. If, instead, it is the single stars that are a small minority, then a binary population with mass-independent parameters (periods, eccentricity, etc.) would produce the same segregation pattern as single-star models (since mean and maximum masses both double, except that $t_{\text {seg }}$ would be even shorter for the same initial mass function as single stars). A little thinking and some tests with random pairing of stars leads to a fraction $\approx 40 \%$ of binaries for an optimal bias in the parameter $\eta$ over time. Kouwenhoven \& de Grijs (2008), Kouwenhoven et al. (2009) and Weidner et al. (2009) have studied this issue quantitatively. These studies indicate that massive clusters (of mass $\sim 10^{6} \mathrm{M}_{\odot}$ ) are robust to a bias in spectrophotometric measures because the velocity dispersion of the binary centres of mass are significantly larger on the mean than the internal dispersion of the binaries. This will break down for low-mass open clusters, since now the internal dispersion is larger than that caused by the cluster's self-gravity.

To pin down these issues more firmly will require a better understanding of cluster properties at birth. Considering that protostars may condense on a timescale not shorter than several $\times 10^{5}$ years, one cannot help but conclude that cluster and star formation censure each other, in the sense that protostars will trace out a significant number of orbits in a dense cluster over their formation timescale. That this will imprint the properties of the cluster (its mass profile and velocity dispersion) as well as the stellar population (binary periods, destruction of loose binaries and the like) is clear, as was shown by Wiersma et al. (2006), who concluded that pre-main-sequence stellar evolution in a cluster led to the depletion of the binary population as well as a shift in its period distribution. Because it is doubtful whether a generic formation scenario applies to all clusters (clumpy accretion for open clusters, e.g., McMillan et al. 2007; near-virial equilibrium throughout the phase of star formation for more massive clusters, e.g., Tan et al. 2006), one may have no choice but to cover as broad a range in initial conditions as possible but aimed at modelling target clusters. Spectacular examples of this are the study by Hurley et al. (2005) for the open cluster M67 using direct-integration $N$-body models, and Giersz \& Heggie (2009) and Heggie \& Giersz (2008) for Monte Carlo models of clusters M4 and NGC 6397. It is well worth stressing here that a winning strategy for treating multiple stars in clusters (binaries, triples, quadruples, and so forth) may be in the form of hybrid codes such as the Monte Carlo approach of Giersz \& Spurzem (2000, 2004 ) which treats complex interactions with direct integration (no assumption on cross sections). The caveat is that at least a few realisations will be needed for a meaningful outcome. An important point which was made for single-star populations, that observables are best derived from light fluxes and not mass, applies here as well in the case of binary and multiple stars: see Hurley (2007) for an application to estimates of the core radius and a revised measure of expansion driven by binaries and intermediate-mass black holes (e.g., Mackey et al. 2008). 


\subsection{Evolved stellar populations: millisecond pulsars, multiple main sequences}

The modelling of multiple stars is important as they open up a large array of collision events that may lead to exotic stellar remnants, such as millisecond pulsars (see, e.g., Ransom 2008 for a review). The demographics of binary and multiple stars have attracted much attention for a number of years, especially as regard their fraction in the dense cores of clusters. This is were physical collisions with stars are more likely to occur. Conventional wisdom would have primordial binaries ejected at high velocities through gravitational recoil and it is worth mentioning once more that stellar interactions would take place already in the protostellar stage of star formation, and that this phase ultimately bears on the statistics of multiple stars (see Wiersma et al. 2006 for binary stars). One would then infer a large fraction of primordial binaries so that at least a few percent survive long enough in the core to form an exotic stellar remnant. It is very exciting to find instead, with Hurley et al. (2007) using $N$-body simulations, that while binaries may be ejected from the core, they may well have centre-of-mass velocities below the cluster's escape velocity. These binaries may then sink back towards the cluster core efficiently, thanks to the shorter segregation time (Equation 3.3), and replenish the core. Overall, the core binary fraction of $\sim 20 \%$ remains nearly constant over time (although the core itself will slowly shrink). I find comfort in the fact that this result was recently confirmed by Fregeau et al. (2009), who rectified their earlier statistics published in Ivanova et al. (2005) using an improved version of their Monte Carlo code. Thus, these two studies and methods independently provide insight into core binary dynamics. This point may appear banal to make, yet it is essential because one finds only partial overlap in the parameter space that the methods each can cover.

To conclude, I should mention another big challenge to dynamicists and stellar evolution experts, which illustrates perfectly the necessity of marrying these two fields and this is the observations of multiple stellar main sequences in rich clusters (for resent updates see, e.g., Piotto et al. 2007 [NGC 288] and Milone et al. 2009a [NGC 1851]). These different main-sequence threads arise from systematic differences in CNO abundances that are difficult to interpret through a universal stellar mass function and require selfenrichment with a flattened high-mass stellar distribution function (d'Antona \& Caloi 2004; but see Pflamm-Altenburg \& Kroupa 2009 for an alternative view). So far, the efficiency of self-enrichment was somewhat difficult to quantify, but recent developments in models of slow winds from rapidly rotating stars (Decressin et al. 2007; d'Ercole et al. 2008) have made it more likely that a standard stellar mass function but with added physics of rotating massive stars strongly suggest that the fine threads are, in fact, more common than could have been anticipated (Milone et al. 2009b), a situation that will keep cluster research buzzing for some time still.

\section{Acknowledgements}

It is a pleasure to thank the organisers of this fine meeting, and R. de Grijs and J. Lépine in particular for the invitation to attend, and both as editors for patiently waiting for this short contribution submitted late.

\section{References}

Aarseth, S. J. 2003, Gravitational N-Body Simulations, Cambridge: Cambridge University Press Aarseth, S. J. \& Heggie, D. C. 1998, MNRAS, 297, 794

Agekian, T. A. 1958, Sov. Astron., 2, 22

Alexander, T. \& Hopman, C. 2009, ApJ, 697, 1861

Allison, R. J., Goodwin, S. P., Parker, R. J., de Grijs, R., Portegies Zwart, S. F., \& Kouwenhoven, M. B. N. 2009, ApJ (Letters), 700, L99 
Amaro-Seoane, P., Gair, J. R., Freitag, M., et al. 2007, Class. Quant. Grav., 24, 113

Ardi, E., Baumgardt, H., \& Mineshige, S. 2008, ApJ, 682, 1195

Bastian, N. \& Goodwin, S.P. 2006, MNRAS (Letters), 369, L9

Bastian, N., Saglia, R. P., Goudfrooij, P., Kissler-Patig, M., Maraston, C., Schweizer, F., \& Zoccali, M. 2006, A\&̈A, 448, 881

Baumgardt, H., De Marchi, G., \& Kroupa, P. 2008, ApJ, 685, 247

Baumgardt, H., Heggie, D. C., Hut, P., \& Makino, J. 2003, MNRAS, 341, 247

Baumgardt, H., Makino, J., Hut, P., McMillan, S., \& Portegies Zwart, S. 2003, ApJ (Letters), $589, \mathrm{~L} 25$

Belleman, R. G., Bédorf, J., \& Portegies Zwart, S. F. 2008, NewA, 13, 103

Berentzen, I., Preto, M., Berczik, P., et al 2009, ApJ, 695, 455

Binney, J. J. \& Tremaine, S. D. 2008, Galactic Dynamics, 2nd ed., Princeton: Princeton University Press

Boily, C. M., Lançon, A., Deiters, S., \& Heggie, D. C. 2005, ApJ (Letters), 620, L27

Cohn, H. N. 1979, ApJ, 234, 1056

D'Antona, F. \& Caloi, V. 2004, ApJ, 611, 871

Decressin, T., Meynet, G., Charbonnel, C., Prantzos, N., \& Ekström, S. 2007, A\&̊A, 464, 1029

Decressin, T., Baumgardt, H., \& Kroupa, P. 2008, A\&A, 492, 101

de Grijs, R., Johnson, R. A., Gilmore, G. F., \& Frayn, C. M. 2002, MNRAS, 331, 228

de Grijs, R. \& Parmentier, G. 2007, ChJAE\&A, 7, 155

D'Ercole, A., Vesperini, E., d'Antona, F., McMillan, S. L. W., \& Recchi, S. 2008, MNRAS, 391, 825

Drukier, G. A., Cohn, H. N., Lugger, P. M., \& Yong, H. 1999, ApJ, 518, 233

Einsel, C. \& Spurzem, R. 1999, MNRAS, 302, 81

Ernst, A., Glaschke, P., Fiestas, J., Just, A., \& Spurzem, R. 2007, MNRAS, 377, 465

Faber, N. T., et al. 2009 MNRAS 2009, in press

Fiestas, J., Spurzem, R., \& Kim, E. 2006, MNRAS, 373, 677

Fiestas, J., Porth, O., \& Spurzem, R. 2008, in: E. Vesperini, M. Giersz, \& A. Sills (eds), Dynamical Evolution of Dense Stellar Systems, Proc. IAU Symp. 246, p. 146

Fleck, J.-J., Boily, C. M., Lançon, A., Deiters, S. 2006, MNRAS, 369, 1392

Fregeau, J. M., Joshi, K. J., Portegies Zwart, S. F., \& Rasio, F. A. 2002, ApJ, 570, 171

Fregeau, J. M., Larson, S. L., Miller, M. C., et al. 2006, ApJ (Letters), 646, L135

Fregeau, J. M., Ivanova, N., \& Rasio, F. A. 2009, ApJ, submitted (arXiv:0907.4196)

Freitag, M., Rasio, F. A., \& Baumgardt, H. 2006a, MNRAS, 368, 121

Freitag, M., Gürkan, M. A., \& Rasio, F. A. 2006b, MNRAS, 368, 141

Fujii, M., Iwasawa, M., Funato, Y., \& Makino, J. 2008, ApJ, 686, 1082

Gaburov, E., Gualandris, A., \& Portegies Zwart, S. 2008, MNRAS, 384, 376

Gaburov, E., Harfst, S., \& Portegies Zwart, S. 2009, NewA, 14, 630

Gebhardt, K., Rich, R. M., \& Ho, L. C. 2005, ApJ, 634, 109

Giersz, M. \& Heggie, D. C. 2009, MNRAS, 395, 1173

Giersz, M. \& Spurzem, R. 1994, MNRAS, 269, 241

Giersz, M. \& Spurzem, R. 2000, MNRAS, 317, 581

Giersz, M. \& Spurzem, R. 2003, MNRAS, 343, 781

Glebbeek, E., Gaburov, E., de Mink, S. E., Pols, O. R., \& Portegies Zwart, S. F. 2009, A\&্A, 497,255

Goodman, J., Heggie, D. C., \& Hut, P. 1993, ApJ, 415, 715

Gürkan, M. A., Freitag, M., \& Rasio, F. A. 2004, ApJ, 604, 632

Hachisu, I. 1979, PASJ, 31, 523

Hemsendorf, M. \& Merritt, D. 2002, ApJ, 580, 606

Hurley, J. R., Pols, O. R., Aarseth, S. J., \& Tout, C. A. 2005, MNRAS, 363, 293

Hurley, J. R. 2007, MNRAS, 379, 93

Hurley, J. R., Aarseth, S. J., \& Shara, M. M. 2007, ApJ, 665, 707

Hut, P. 2003, in: Globular Clusters: Formation, Evolution and the Role of Compact Objects (astro-ph/0309395) 
Hut, P. \& Heggie, D. C. 2002, J. Stat. Phys., 109, 1017

Ivanova, N., Belczynski, K., Fregeau, J. M.,\& Rasio, F. A. 2005, MNRAS, 358, 572

Kim, E., Einsel, C., Lee, H. M., Spurzem, R., \& Lee, M. G. 2002, MNRAS, 334, 310

Kim, E., Lee, H. M., \& Spurzem, R. 2004, MNRAS, 351, 220

Kim, E., Yoon, I., Lee, H. M., \& Spurzem, R. 2008, MNRAS, 383, 2

Kong, A. K. H. 2007, ApJ, 661, 875

Kouwenhoven, M. B. N., Brown, A. G. A., Goodwin, S. P., Portegies Zwart, S. F. \& Kaper, L. 2009, A\&BA, 493, 979

Kouwenhoven, M. B. N., \& de Grijs, R. 2008, A\&A, 480, 103

Louis, P. D. \& Spurzem, R. 1991, ApJ, 251, 408

Lynden-Bell, D. \& Eggleton, P. E. 1980, MNRAS, 191, 483

Mackey, A. D., Wilkinson, M. I., Davies, M. B., \& Gilmore, G. F. 2008, MNRAS, 386, 65

Makino, J. 1996, ApJ, 471, 796

Makino, J. \& Aarseth, S. J. 1992, PASP, 44, 141

McCrady, N. \& Graham, J. R. 2007, ApJ, 663, 844

McCrady, N., Graham, J. R., \& Vacca, W. D. 2005, ApJ, 621, 278

McMillan, S. L. W. 1986, ApJ, 307, 126

McMillan, S. L. W., Vesperini, E., \& Portegies Zwart, S. F. 2007, ApJ (Letters), 655, L45

Merritt, D. 2006, Rep. Prog. Phys., 69, 2513

Merritt, D., Gualandris, A., \& Mikkola, S. 2009, ApJ, 693, 35

Meylan, G. \& Heggie, D. C. 1997, A\&ARev., 8, 1

Miller, R. H. 1971, J. Comput. Phys., 8, 449

Milone, A. P., Stetson, P. B., Piotto, G., Bedin, L. R.; Anderson, J., Cassisi, S., \& Salaris, M. 2009b, A\&A, 503, 755

Milone, A. P., Bedin, L. R., Piotto, G., \& Anderson, J. 2009a, A\&A, 497, 755

Noyola, E. \& Gebhardt, K. 2006, AJ, 132, 447

Östlin, G., Cumming, R. J., \& Bergvall, N. 2007, A\&A, 461, 471

Pflamm-Altenburg, J. \& Kroupa, P. 2009, MNRAS, 397, 488

Piotto, G., Bedin, L. R., Anderson, J., King, I. R., Cassisi, S., Milone, A. P., Villanova, S., Pietrinferni, A., \& Renzini, A. 2007, ApJ (Letters), 661, L53

Pooley, D. \& Rappaport, S. 2006, ApJ (Letters), 644, L45

Portegies Zwart, S. F. \& McMillan, S. L. W. 2002, ApJ, 576, 899

Portegies Zwart, S. F., McMillan, S. L. W., \& Gerhard, O. 2003, ApJ, 593, 352

Portegies Zwart, S. F., Baumgardt, H., Hut, P., et al. 2004, Nature, 428, 724

Portegies Zwart, S., McMillan, S., Harfst, S., et al. 2009, NewA, 14, 369

Ransom, S. M. 2008, in: E. Vesperini, A. Sills, \& M. Giersz (eds.), Dynamical Evolution of Dense Stellar Systems, Proc. IAU Symp. 246, p. 291

Richtler, T. \& Larsen, S. 2009, Globular Clusters: Guides to Galaxies, ESO Astrophys. Symp. Ser., Heidelberg: Springer

Schive, H.-Y., Chien, C.-H., Wong, S.-K., Tsai, Y.-C., \& Chiueh, T. 2008, NewA, 13, 418

Shapiro, S. L. \& Marchant, A. B. 1976, ApJ, 210, 757

Spitzer Jr., L. 1969, ApJ (Letters), 158, L139

Spitzer Jr., L. 1987, Dynamics of Star Clusters, Princeton: Princeton University Press

Spurzem, R., Giersz, M., Takahashi, K., \& Ernst, A. 2005, MNRAS, 364, 948

Takahashi, K. 1995, PASJ, 47, 561

Tan, J. C., Krumholz, M. R., \& McKee, C. F. 2006, ApJ, 641, L121

Vazdekis, A. \& Peletier, R. F. 2007, Stellar Populations as Building Blocks of Galaxies, Proc. IAU Symp. 241 Cambridge: Cambridge University Press

Vesperini, E., McMillan, S. L. W., \& Portegies Zwart, S.F. 2009, ApJ, 698, 615

Vesperini, E., Giersz, M., \& Sills, A. 2008, Dynamical Evolution of Dense Stellar Systems, Proc.

IAU Symp. 246 Cambridge: Cambridge University Press

Weidner, C., Kroupa, P., \& Maschberger, T. 2009, MNRAS, 393, 663

Wiersma, R., Sills, A., \& Portegies Zwart, S. F. 2006, ApJ, 637, 838 